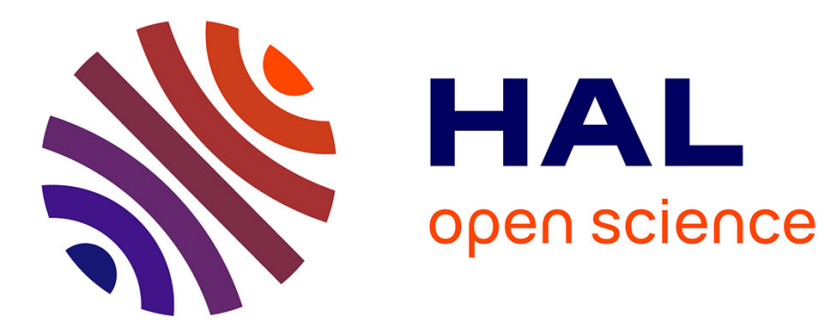

\title{
NanoRobotic Structures with Embedded Actuation via Ion Induced Folding
}

\author{
Amine Benouhiba, Léo Wurtz, Jean-Yves Rauch, Joel Agnus, Kanty \\ Rabenorosoa, Cédric Clevy
}

\section{To cite this version:}

Amine Benouhiba, Léo Wurtz, Jean-Yves Rauch, Joel Agnus, Kanty Rabenorosoa, et al.. NanoRobotic Structures with Embedded Actuation via Ion Induced Folding. Advanced Materials, 2021, 33 (45), pp.2103371. hal-03455032

\section{HAL Id: hal-03455032 \\ https://hal.science/hal-03455032}

Submitted on 26 Jan 2022

HAL is a multi-disciplinary open access archive for the deposit and dissemination of scientific research documents, whether they are published or not. The documents may come from teaching and research institutions in France or abroad, or from public or private research centers.
L'archive ouverte pluridisciplinaire HAL, est destinée au dépôt et à la diffusion de documents scientifiques de niveau recherche, publiés ou non, émanant des établissements d'enseignement et de recherche français ou étrangers, des laboratoires publics ou privés. 


\section{NanoRobotic Structures with Embedded Actuation via Ion In- duced Folding}

Amine Benouhiba, Léo Wurtz, Jean-Yves Rauch, Joël Agnus, Kanty Rabenorosoa, and Cédric Clévy FEMTO-ST Institute, Univ. Bourgogne Franche-Comté, CNRS

AS2M department

24 rue Alain Savary, 25000 Besançon, France

Email: [amine.benouhiba, jyves.rauch, joel.agnus, rkanty, cclevy]@femto-st.fr

Keywords: ion-induced folding, focused ion beam, $4 D$ structures, nanorobotics, vacuum environment.

4D structures are tridimensional structures with time-varying abilities that provide high versatility, sophisticated designs, and a broad spectrum of actuation and sensing possibilities. The downsizing of these structures below $100 \mu \mathrm{m}$ opens up exceptional opportunities for many disciplines, including photonics, acoustics, medicine, and nanorobotics. However, it requires a paradigm shift in manufacturing methods, especially for dynamic structures. A novel fabrication method based on ion-induced folding of planar multilayer structures embedding their actuation is proposed - the planar structures are fabricated in bulk through batch microfabrication techniques. Programmable and accurate bidirectional foldings $\left(-70^{\circ}-+90^{\circ}\right)$ of $\mathrm{SiO}_{2} / \mathrm{Cr} / \mathrm{Al}$ multilayer structures are modeled, experimentally demonstrated then applied to embedded electrothermal actuation of controllable and dynamic 4D nanorobotic structures. The method is used to produce high-performances case-study grippers for nanorobotic applications in confined environments. Once folded, a gripping task at the nano-scale is demonstrated. The proposed fabrication method is suitable for creating small-scale $4 \mathrm{D}$ systems for nanorobotics, medical devices, and tunable metamaterials, where rapid folding and enhanced dynamic control are required.

As an emerging research field, nanorobotics has opened new avenues for numerous applications, such as nanomanipulation, assembly, manufacturing, precision surgery, drug delivery, medical diagnosis, detoxification, and personalized medicine $[1,2,3]$. Despite the promising potential of nanorobotics, it still relies heavily on the traditional planar microfabrication technology. Therefore, the development of nanosystems remains subject to significant technical and physical restrictions in terms of design, functionality, and dexterity. A few methods have been developed for the fabrication of $4 \mathrm{D}$ nanosystems for a variety of applications, including nanorobotic systems in particular. However, fabricating 4D nanorobotic systems with accurate morphology, multiple materials, and a high dynamic behavior is an extremely challenging task. At this scale, the fabrication of $4 \mathrm{D}$ structures remains one of the main challenges for reaching the next generation of nanorobotic systems.

In the last decade, tridimensional microfabrication has been experiencing rapid growth, and few methods have emerged, such as layer by layer stacking $[4,5,6]$, direct laser writing $[7,8,9,10]$, template synthesis $[11,12,13]$, and self-assembly [14, 15, 16]. Among these methods, Focused Ion Beam Stress-Induced Deformation (FIB-SID) have shown great promises thanks to its ability to turn planar thin films into sophisticated volumetric structures by folding $[17,18,19]$. Unlike other techniques, and as shown in [20], FIB-SID is a one-step process that can generate tridimensional structures with a nano-scale precision and using various materials (dielectric, semiconductor, and thin metal films). As a result, FIB-SID, with its ability to go from simple planar design to highly sophisticated volumetric structures by folding, holds great promise. This process has been used for the fabrication of many nanosystems, from simple folded beam structures [21,22,23], carbon nanotubes [24], and semiconductor nanowires [25] to origami cubes [26, 27], helix [20], metamaterials [28, 29, 30], and kirigami devices [31, 32, 33]. To the best of our knowledge, Mao et al. have firstly proposed actuated structures fabricated by FIB-SID and consist of folding bilayer Si3N4/Au composite, which can be electrothermally activated in a binary status (ON-OFF) in order to control the resonance absorption of a tunable metasurface [29]. Nonetheless, most of these examples are passive/static structures made from a single thin layer. Advanced nanorobotic systems require versatility, highly sophisticated designs, and multi-Degrees of Freedom (multi-DoF). Therefore, having a dynamic behavior is a priority for such systems to be able to act, sense and execute different tasks. In other words, in order for FIB-SID concepts to be beneficial for nanorobotics (among other applications), they must be used for the development of active/dynamic devices, also known as 4D structures where the shape of the structure is spatially and temporally controlled.

We propose a new fabrication method for $4 \mathrm{D}$ dynamic nanorobotic systems through programmable folding and electrothermal actuation. This method is based on FIB-SID folding of multilayer structures ob- 


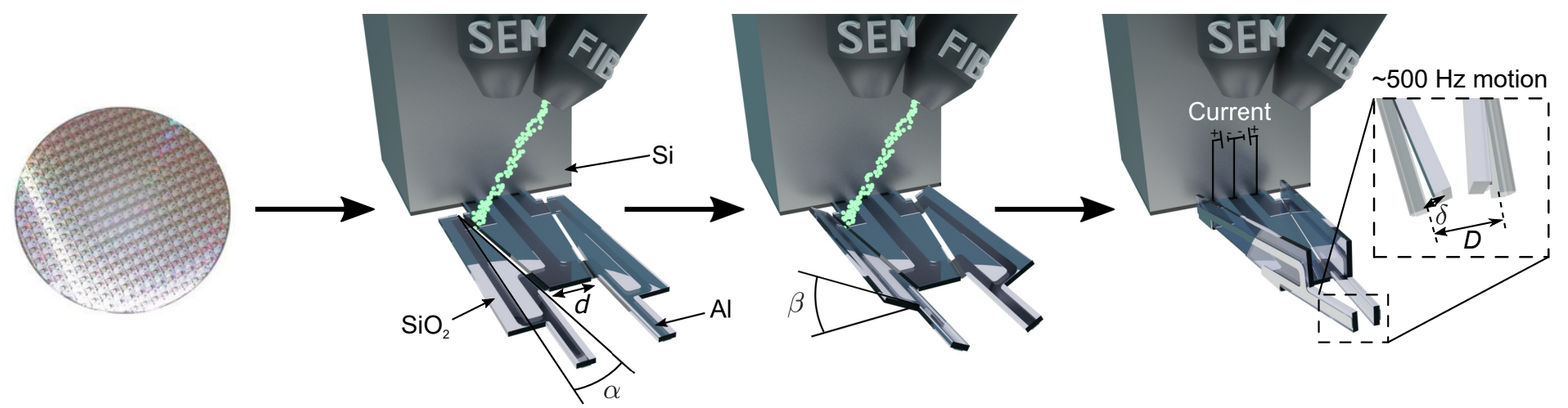

Batch fabrication

2D multi-layer design
FIB-based Patterning
FIB-SID

folding
Personalized dynamic nanorobotic

systems with embeded actuation (gripper)

Figure 1: Proposed 4D fabrication approach for nanorobotic systems, applied to a folded gripper. Batch microfabrication based on planar designs, followed by FIB-based patterning, bi-directional FIB-SID folding, and electrothermal actuation and control of $4 \mathrm{D}$ dynamic folded structures with embedded actuation.

tained through batch microfabrication techniques, as shown in Figure 1. A batch fabrication of planar structures can be produced through the use of the cleanroom technology. Then, depending on the ion-induced folding parameters (patterning location/orientation and folding direction/angle), personalized nanorobotic systems can be fabricated from a planar structure. In this article, the proposed planar systems contain three different layers, structured as follows: first, a structural layer that gives the nanorobotic systems its geometrical and mechanical properties (thick dielectric layer). Then, an active layer that induces the FIB-SID folding allows the nanorobotic systems' actuation thanks to a Joule heating controlled bending caused by the differential thermal expansion between the two layers (thin metal layer). Finally, a seed layer that ensures an adherence between the two first layers (a very thin layer of Chromium). Once the planar structures are produced, the ion-induced folding can be achieved following two steps. The first step is patterning, also known as the removal of the structural and seed layers. The active layer is revealed in specific locations and with specific orientations thanks to the use of FIB etching. The patterning is one of the key steps which determines the final properties of the nanorobotic system. The patterning operation is then followed by the folding one. Unlike the first two layers, the FIB etching of the active layer leads to stress-induced deformations, which results in folding. Depending on the parameters of the FIB etching (ion dose), of the used material and its thickness, the folding can be bidirectional and programmable (from $-70^{\circ}$ to $+90^{\circ}$ ). It was demonstrated (as shown in Figure 2) that at first, the folding goes downward, and once the folding angle $\beta$ reaches its maximum downward folding $\left(\beta=-70^{\circ}\right)$, it starts going upward all the way to its maximum $\left(\beta=+90^{\circ}\right)$. The proposed fabrication method offers versatility and many possibilities in terms of planar structures, materials selection (insulators and metals), patterning strategies, and folding angles, making the desired sophisticated 4D structures highly achievable. As a result, a wide range of $4 \mathrm{D}$ nanorobotic systems can be realized/achieved. Lastly, the folded 4D nanorobotic system can be dynamically controlled through Joule heating-induced actuation.

In order to showcase the efficiency of the proposed fabrication method, we designed, modeled, fabricated, and characterized a monolithic gripper as a proof of concept. The planar design of the proposed gripper has dimensions of $77 \times 28 \mu \mathrm{m} \times \mu \mathrm{m}$. It contains three layers: a $1.2 \mu \mathrm{m}$ thick Silica $\left(\mathrm{SiO}_{2}\right)$ structural layer, a $200 \mathrm{~nm}$ thick $A l$ active layer, and a $20 \mathrm{~nm}$ thick Chromium $(\mathrm{Cr})$ seed layer. The batch fabrication of the planar design is easily achieved with a three-mask process, as we use a single thin layer of $\mathrm{Al}$ to simultaneously act as the active layer during folding, as the electrothermal heater during actuation, and as the electric circuit for control. For the patterning of the gripper, an ion dose of $6.2 \times 10^{+24}\left(\mathrm{ion} / \mathrm{cm}^{2}\right)$ is used to reveal the $\mathrm{Al}$ active layer, by the process of etching the $\mathrm{SiO}_{2}$ and the $\mathrm{Cr}$ layers. The patterning is represented by two long strips separated by a $5 \mu \mathrm{m}$ gap (named as $d$, see Figure 1 ), and both strips have an inclination of $6^{\circ}$, also known as the patterning angle $\alpha$. One with an $\alpha$ of $+6^{\circ}$, and the 


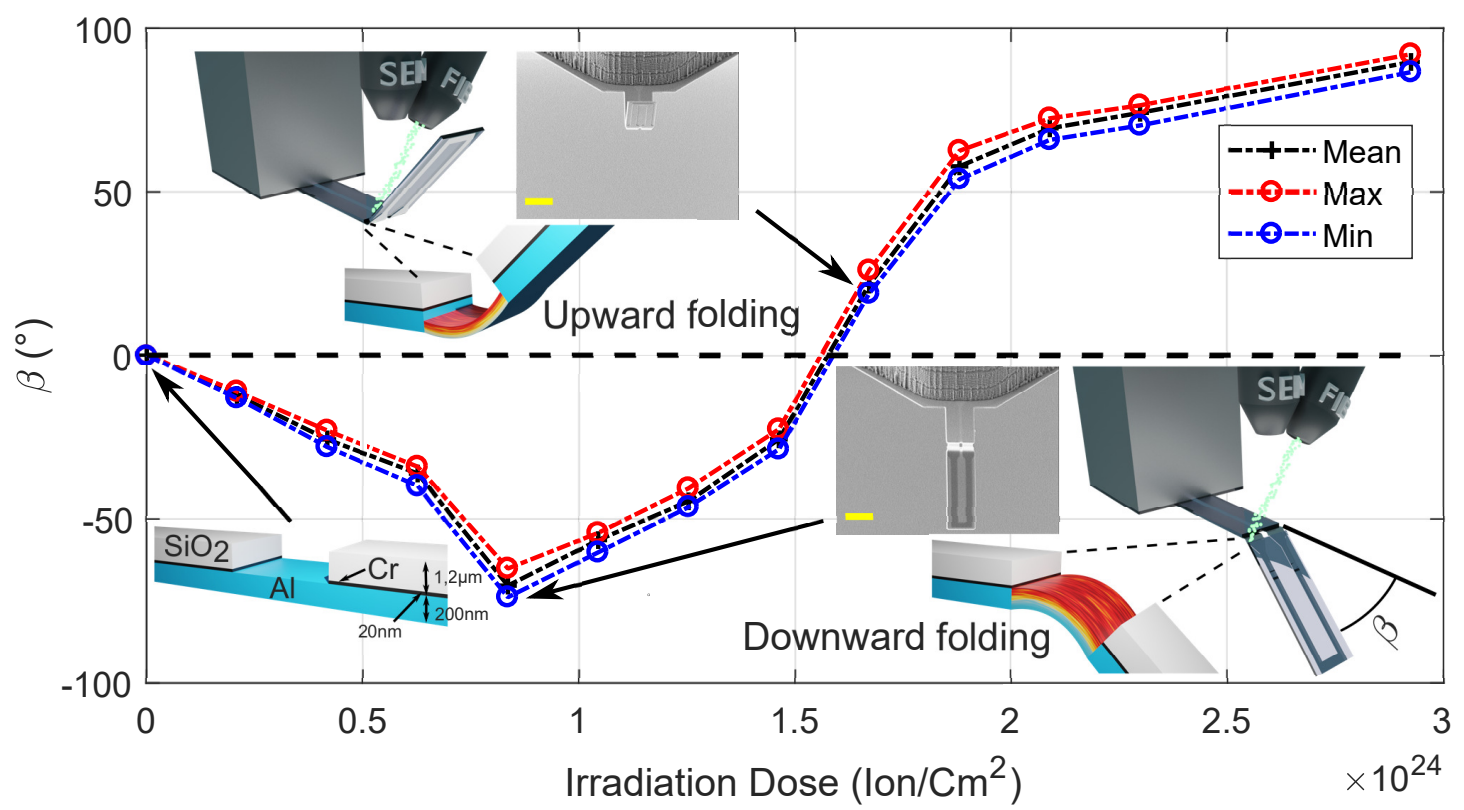

Figure 2: Programmable bidirectional FIB-SID folding behavior of multi-layer $\left(\mathrm{SiO}_{2} / 1.2 \mu \mathrm{m}, \mathrm{Cr} / 20 \mathrm{~nm}\right.$, and $\left.\mathrm{Al} / 200 \mathrm{~nm}\right)$ cantilever in function of ion irradiation dose. At low ion doses, the cantilever folds downward $\left(\beta=-9.5^{\circ}\right.$ for an ion dose of $0.2 \times 10^{24}$ ion $\left./ \mathrm{cm}^{2}\right)$, until it reaches its maximum downward folding angle $\left(\beta=-70^{\circ}\right)$, for an ion dose of $0.84 \times 10^{24}$ ion $/ \mathrm{cm}^{2}$. Any further irradiation, in this case, will lead to an upward folding, which first passes by the initial shape of the structure (unfolded cantilever) and continues folding to reach an angle $\beta$ of $+68^{\circ}$ for an ion dose of $2.1 \times 10^{24}$ ion $/ \mathrm{cm}^{2}$ ). Due to the multilayer aspect of the structure, folding becomes relatively slower after this point, because the more the structures folds, the more the irradiation of the $\mathrm{Al}$ active layer is obstructed, until being totally obstructed at a $\beta=+90^{\circ}$. This value corresponds to an ion dose of $2.9 \times 10^{24} \mathrm{ion} / \mathrm{cm}^{2}$. The measurement uncertainties are included in the curve, and the ion beam current and acceleration voltages used for the folding are $10 \mathrm{nA}$ and $30 \mathrm{keV}$, respectively. Scale bars of the SEM images $=20 \mu m$.

other with an $\alpha$ of $-6^{\circ}$ (as shown in Figure 3). Each of the strips has dimensions of $65 \times 1.4 \mu \mathrm{m} \times \mu \mathrm{m}$. The characteristics of the produced gripper can be controlled through the patterning parameters. For instance, by varying the patterning angle $\alpha$, the initial gap between the gripper's fingers can be set to different values (as shown in Figure 3). This is also true for the space between the two patterning strips $d$. Therefore, using the same planar design, different personalized grippers with different properties and ranges of manipulation can be created. In other words, the properties of the nanorobotic system are customizable throughout the patterning parameters. For example, the black dashed line in Figure 3 shows the decrease in the gripper opening $D$, produced by increasing the patterning angle $\alpha$. This will allow the gripper to handle smaller and smaller objects as it is operated (the red lines in Figure $\mathbf{3}$ give the range of the tip displacement). The tip displacement of the folded gripper is experimentally characterized and show the continuous motion of the finger's tip displacement (Figure 5a-d). Each finger has a maximum tip displacement of $1.1 \mu \mathrm{m}$, which corresponds to a maximum input current I of $14 \mathrm{~mA}$. After the FIB patterning, the ion-induced folding of the gripper is investigated (see Figure 4). From a foldability standpoint, the planar structure maintains its initial state (unfolded state) during the patterning (see Figure 4a). Therefore, the ion irradiation parameters used for the patterning (ion beam current and acceleration voltage) do not directly affect the folding afterwards. However, low ion beam currents, in this case, will result in a better etching profile. The latter enables better FIB irradiation of the active layer (thanks to the better revealing of the layer), which subsequently leads to a better folding process. The $200 \mathrm{~nm}$ Al layer serve as hinges, bending means during ion irradiation, and final electrode for the activation of the gripper fingers (see Figure 4b). Moreover, depending on the folding direction, the produced gripper provides different gripping characteristics. For instance, upward folding (downward folding) generates a naturally open (naturally closed) gripper that closes (opens) when actuated. To summarize, the grippers are held by a silicon support and are made by three materials: a thin layer 

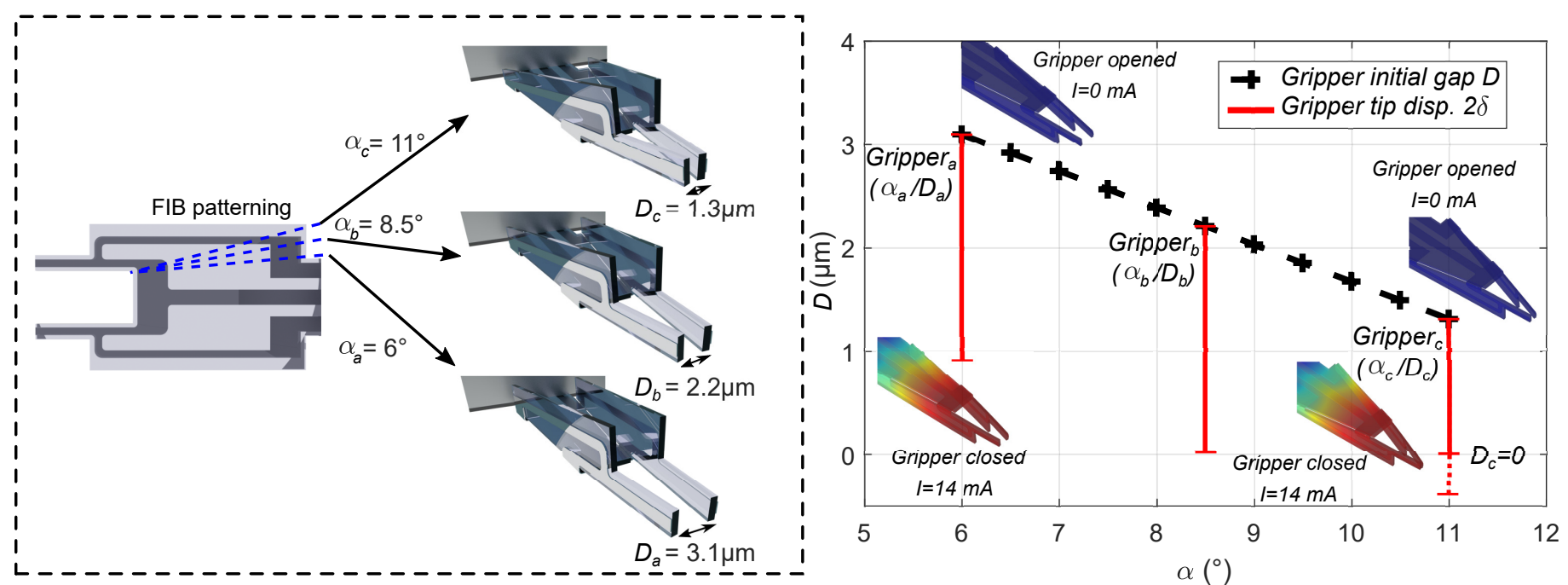

Figure 3: Gripper's initial gap and the range of its fingers' tip displacement vs. the patterning lines. Depending on the value of the patterning angle $\alpha$, the initial gap $D$ of the gripper can be adjusted, while the fingers' tip displacement remains unaffected; therefore, the properties of the gripper can be tailored to the desired range size of the manipulated objects. The red line highlights the change in this gap resulting from the actuation of the gripper $(2 \delta)$, and the red dotted line represents a completely closed gripper (generated contact force).

of silica of $1.2 \mu \mathrm{m}$, a $\mathrm{Cr}(20 \mathrm{~nm})$ is deposited as a seed layer followed by a $200 \mathrm{~nm}$ thick Al layer. All these materials support a standard use in a vacuum environment, but if the application requires other working environments (liquid for instance), it is possible to choose other materials, dimensions or functionalize the surfaces. Depending on the needed characteristics (high temperature, biocompatibility, basic solution i.e. $\mathrm{pH}>11$ etc.), Ti, Pt, Au, etc., can be used. Further details on the ion irradiation parameters that control the patterning and folding processes, as well as the folding mechanism, are given in Section I of the supporting information.

a)

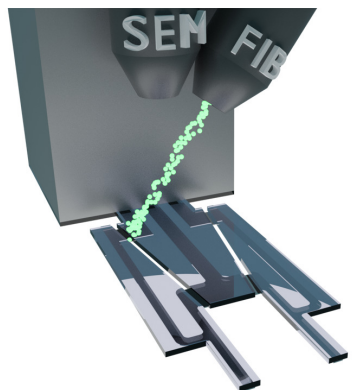

b)

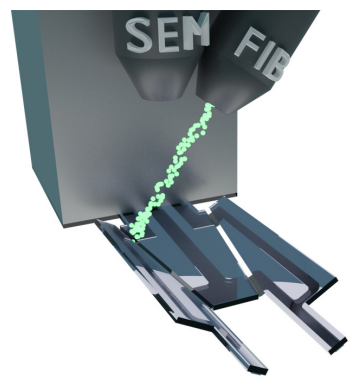

c)

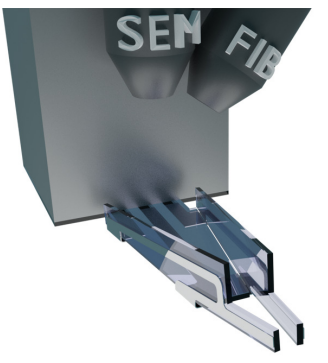

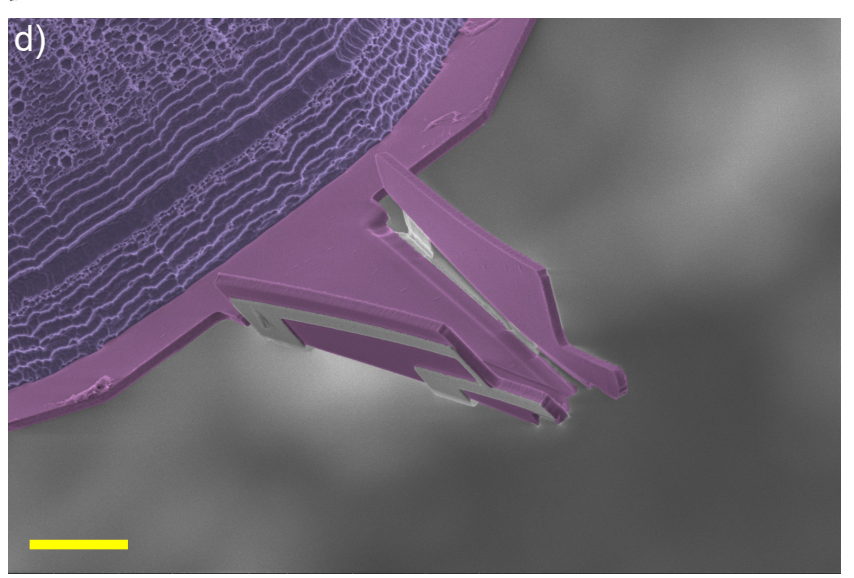

Figure 4: FIB-SID folding of the gripper. a-c) Illustration of the folding of the gripper, a) patterning (removal of the passive layers), b) ion-induced folding at $60^{\circ}$, and c) at $90^{\circ}$. d) colored image of a bottom SEM view of a folded gripper. Scale bar $=10 \mu m$. 
a)

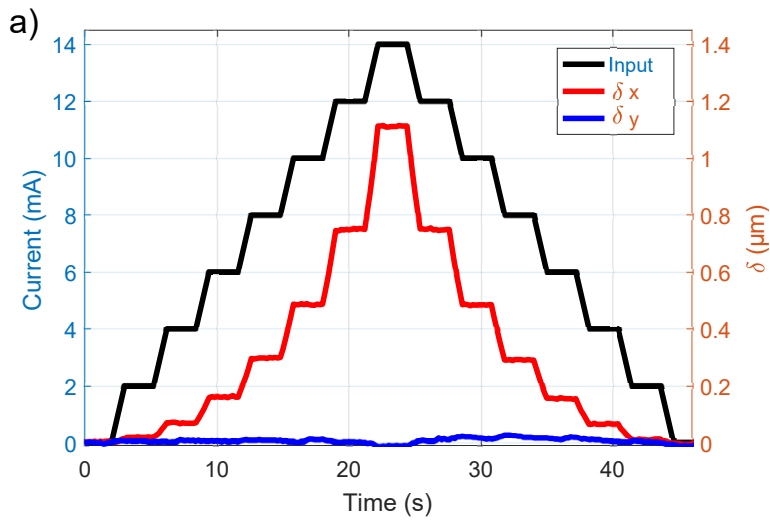

c)
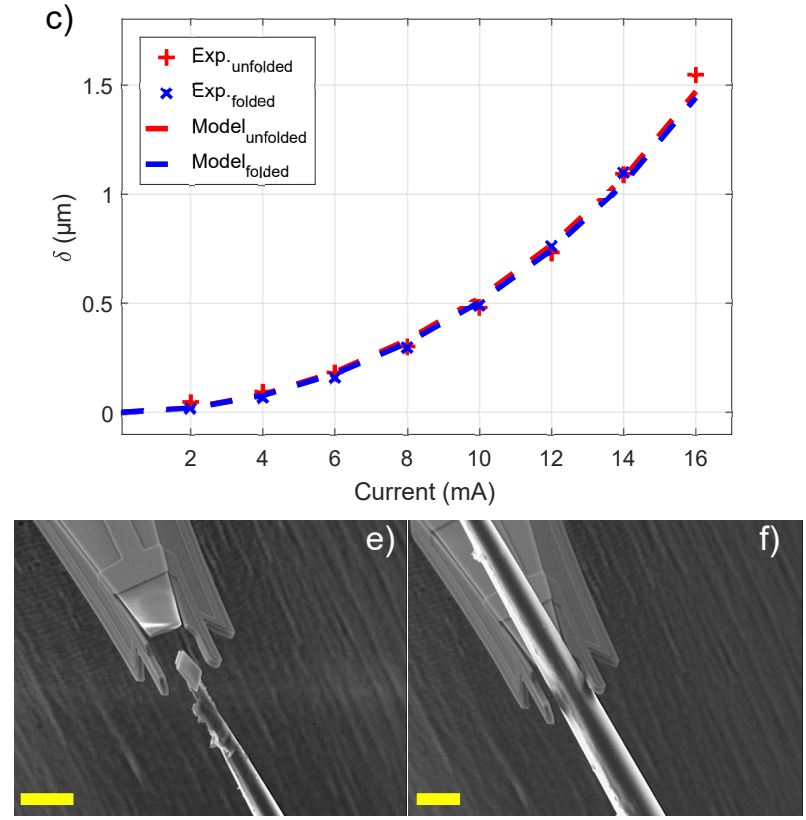

b)

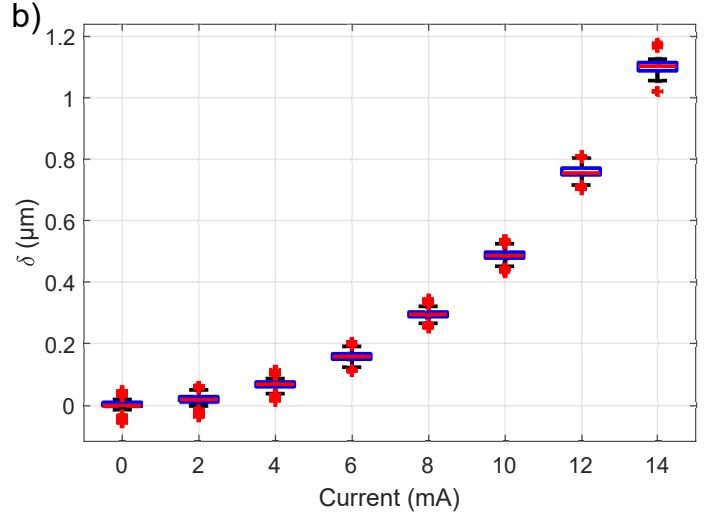

d)
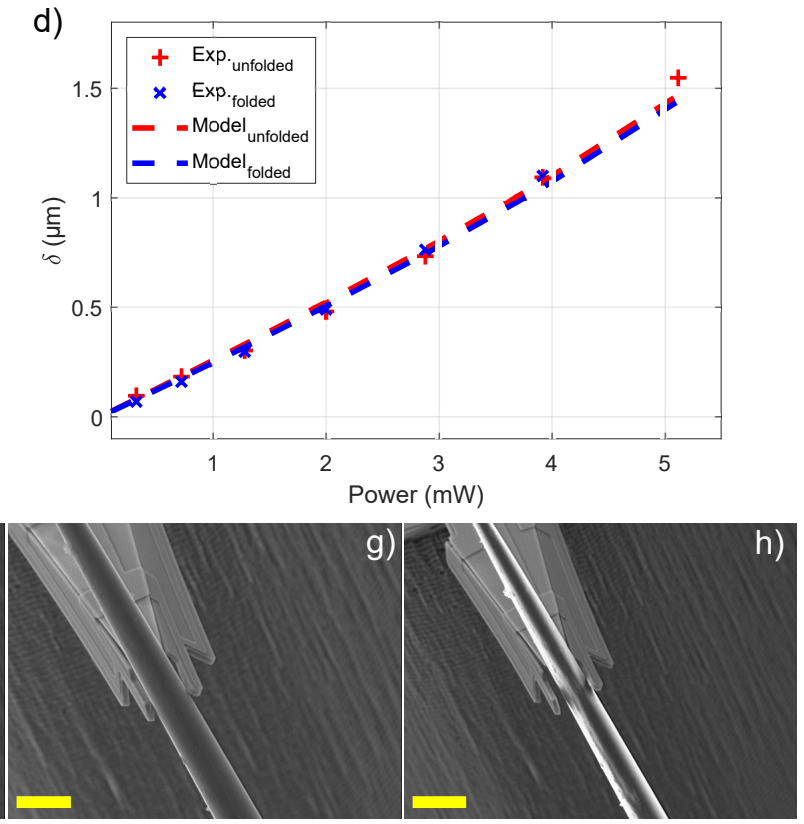

Figure 5: Actuation, characterization, and comparison between an unfolded and folded gripper. a) finger's tip displacement of a folded gripper, in response to a step stairs input signal $(1.1 \mu \mathrm{m}$ maximum displacement for a maximum input current of $14 \mathrm{~mA}$ ). b) statistical results for three folded grippers tested five times each. c) and d) continuous displacement evolution of the gripper tip versus the applied current, this Figure also includes the comparisons of the average tip displacement of unfolded and folded grippers, d) comparison between the average finger's tip displacement of unfolded and folded grippers and their respective FE models depending on the current and power input signals, respectively. Regarding unfolded grippers results, only deformations produced by the fingers are considered here. $\mathrm{d}-\mathrm{g}$ ) Sequence of images during the interaction with an optical fiber's tip: d) locating, e) approaching, f) gripping, and g) releasing of the object. Scale bars $=10 \mu m$.

In order to assess the efficiency of the fabrication process, the grippers are characterized before and after folding. The performances of both states are then evaluated. Additionally, to design and better understand the behavior of the fabricated device, a Finite Element (FE) modeling is used. The model describes the behaviors of the grippers before, as well as, after folding. The software COMSOL Multiphysics used for the numerical analysis (as shown in Figure 5c-d) is coupled with Matlab software through the Livelink interface to allow for parameters' identification. The identification process is based on a genetic algorithm. Additionally, the model is also used to conduct a time-dependent analysis of the gripper's behavior, which permits the calculation of the system's cutoff frequency at $500 \mathrm{~Hz}$. To study the behavior of the grippers (tip displacement) to an input signal (current), online visual tracking is used. The visual tracking of the grippers tip displacement is done using a normalized cross-correlation technique based on the SEM images. First, the tip displacement of unfolded grippers is measured in response to an input step stairs signal, with a minimum current value of $2 \mathrm{~mA}$, a maximum current value of $16 \mathrm{~mA}$, and a step increment in the current value of $2 \mathrm{~mA}$. The maximum value of the current is fixed at $16 \mathrm{~mA}$ in order to avoid damaging the gripper. In Addition, the steps have a slope of $1 \mathrm{~s}$. Three unfolded grip- 
pers were tested five times each. The results of the tests can be found in Section II of the supporting information. The mean maximum tip displacement measured, which corresponds to the maximum current used $(16 \mathrm{~mA})$, is $2.7 \mu \mathrm{m}$. In the same manner, three folded grippers were tested. The maximum current value, in this case, was fixed at $14 \mathrm{~mA}$. According to Figure 5a-b, the mean maximum tip displacement is $1.1 \mu \mathrm{m}$, which corresponds to an input current value of $14 \mathrm{~mA}$. The experimental results for both cases were used for parameters' identification for the FE model. Consequently, the nanorobotic system is able to maintain its performances after the FIB-SID folding process. This observation is also verified when only the finger deformation is considered for both unfolded and folded cases (see Figure 5cd), where both states of the system outputs the same performances. This outcome confirms that the gripper is able to preserve its performances after the ion-induced folding. It is worth mentioning that each of the three folded grippers was operated over 130 times. Moreover, they were able to maintain their full performances throughout the testing period (no quantifiable degradation). This reproducibility validates the overall approach as well as the fabrication process's effectiveness. Furthermore, from the 130 tests conducted for each gripper, we were able to determine that the folded grippers have a repeatability of less than $20 \mathrm{~nm}$, or less than $2 \%$ of the maximum displacement. The repeatability and scalability of this technology are mainly hinged on the repeatability and scalability of the fabricated planar structures; consequently, we can conclude that this technology is highly scalable and repeatable. A more in-depth characterization of the gripper, before and after folding, that highlights the comparison between the two states is provided in Section II of the supporting information.

Furthermore, to demonstrate the folded gripper's abilities to hold and interact with objects, two different tests are conducted, an interaction with a conductive object (metalized optical fiber's tip) and a dielectric object (small $\mathrm{SiO}_{2}$ block). First, to observe how the gripper interacts during contact with a conductive object (minimal electrostatic charges), the closing action and reopening of the gripper are tested using the metalized optical fiber's tip (as shown in Figure 5f-g). The gripper is able to approach, hold and release the optical fiber's tip with ease and in a smooth fashion. The electrostatic charges, in this case, do not affect the operation of the gripper. Secondly, during a similar test with a small $\mathrm{SiO}_{2}$ block (notable electrostatic charges), the gripper is able to approach, hold and release the object. Compared to the first test, electrostatic charges are apparent, as they slightly delay the release. Nevertheless, the gripper is able to hold and release the object with ease (see Movie S5). Finally, we can conclude that the folded gripper acts effectively in scenarios that include mild as well as notable electrostatic interactions. In this article, we have demonstrated that 4D nanorobotic structures based on embedded electrothermal actuation can be successfully fabricated and actuated to accomplish a robotic task. The proposed fabrication method will open new horizons in nanorobotics and enable the fabrication of other nanosystems for the wealth of nanoengineering. Primarily, by tailoring the patterning and folding parameters, highly sophisticated 4D nanosystems with custom characteristics could be achieved simply using the same planar structure. Additionally, the characteristics can further be tailored by acting on the planar design and/or exploiting the selection of materials that can be used. Moreover, the tethered aspect, in this case, allows for a direct and dynamic $(500 \mathrm{~Hz})$ controllability during advanced function execution and in different vacuum environments. We also envision that the combination of 4D FIB-SID fabricated structures with other actuation techniques, such as light, magnetic, acoustic, etc., will strongly impact the field of nanorobotics in particular and nanoengineering in general. The fabrication of 4D structures via ion-induced folding is an attractive method for producing nanorobotic systems for nanoassembly, manufacturing, and CNTs manipulation in vacuum which can be extended to biomedical applications by considering some design modifications.

\section{Materials and Methods}

\section{$1.1 \quad$ FE modeling}

The numerical analysis for this study is performed using a FE model. The commercial software COMSOL Multiphysics is used to solve the linear elastic deformation equations caused by joule heating (thermal expansion). For the bending calculations, the response of the geometries to the changes in current 
is introduced in the form of a volumetric stress. Three different materials are used for the model, Silica $\left(\mathrm{SiO}_{2}\right)$, Aluminum $(\mathrm{Al})$, and Silicon $(\mathrm{Si})$. Since $\mathrm{Si}$ is only a support layer (base) used to hold the grippers, the parameters used for its modeling are not modified and have the same values provided by the software material library (Young's modulus of $E_{S i}=131 \times 10^{9} \mathrm{~Pa}$ and thermal expansion coefficient $\left.\alpha_{S i}=4.15 \times 10^{-6} 1 / \mathrm{K}\right)$. However, for the two remaining materials, a genetic algorithm based on COMSOL Multiphysics / Matlab coupling (Livelink interface) is used for parameters identification. The identified parameters have values of $E_{\mathrm{SiO}_{2}}=73.1 \times 10^{9} \mathrm{~Pa} / \alpha_{\mathrm{SiO}_{2}}=0.25 \times 10^{-6} 1 / \mathrm{K}$ and $E_{A l}=70 \times 10^{9} \mathrm{~Pa}$ $/ \alpha_{A l}=23.8 \times 10^{-6} 1 / \mathrm{K}$, for $\mathrm{SiO}_{2}$ and $\mathrm{Al}$, respectively. Poisson's ratios of $\nu_{\mathrm{Si}}=0.27, \nu_{\mathrm{SiO}_{2}}=0.17$, and $\nu_{A l}=0.33$ are used for the modeling of $\mathrm{Si}, \mathrm{SiO}_{2}$, and $\mathrm{Al}$, respectively. The same identical parameters are used for the modeling of the different configurations of the grippers, including unfolded and folded states. The outer boundaries of the base were mechanically constrained and were set to room temperature. The element type for the discretization were set to free tetrahedral. Additionally, a time-dependent analysis is also conducted using this model to study the dynamic behavior of the grippers. The system's bode diagram is determined, and the cutoff frequency is identified at $-3 \mathrm{~dB}$.

\subsection{Microfabrication process}

An initial wafer of $\mathrm{SiO}_{2}-\mathrm{Si}-\mathrm{SiO}_{2}$ is used for the batch fabrication of the planar designs. First, the $\mathrm{SiO}_{2}$ is removed from the back of the wafer. Next, on top of the $1.2 \mu \mathrm{m}$ thick $\mathrm{SiO}_{2}$, a lift-off process is performed for the deposition of the electrodes $\mathrm{Cr} / \mathrm{Al}$, with thicknesses of $20 / 200 \mathrm{~nm}$, respectively. The back surface opening ( $\mathrm{Si}$ etching) is conducted using DRIE followed by a front etching of $\mathrm{SiO}_{2}$. Finally, the sample release is executed.

\subsection{Folding of the origami gripper}

The FIB-SID folding is conducted using the $\mu$ Robotex station. The latter is based on an Auriga 60 microscope produced by Zeiss ${ }^{1}$. It contains a large cubic vacuum chamber with dimensions of $60 \times 60 \times 60$ $\mathrm{cm}^{3}$ and SEM field emission gun column vertically installed and accompanied by two detectors for retro diffused electrons: an energy selective backscattered detector and an in-lens detector. A third detector, SE2 detector, is used for the detection of secondary electrons. Furthermore, the FIB column, installed at $54^{\circ}$ angle from the SEM column, is produced by Orsay-Physics ${ }^{2}$. The general settings used for the folding are as follows: an overlap of $50 \%$, a dwell time of $1 \mu \mathrm{s}$, and ion beam acceleration voltage of $30 \mathrm{KeV}$. Additionally, beam currents with a value of $10 \mathrm{nA}$ is used for the patterning and the folding. All the ion doses given in this paper are calculated using the beam current, surface of the irradiated area, and irradiation time. The images presented in this work are obtained using both the SEM and FIB modes. The online tracking of the tip displacement of the grippers is performed using the SEM mode. Lastly, the calculations of the folding angles are done using FIB mode and by considering the viewing angle.

\subsection{Gripper displacement tracking}

The SEM visual tracking of the gripper's tip (unfolded or folded), is managed by MATLAB / Simulink software. It uses a Visual Servoing Platform (ViSP) in addition to a dedicated block set Cvlink ${ }^{3}$. The tip tracking is based on normalized cross-correlation as previously done in [34]. The user selects a small window of frame, which will be tracked online and represented by a red cross in the frame. The tip displacement is measured in pixels before being converted in real-time to micrometers and given as an output. Furthermore, a multi-function data acquisition module (USB-6343) from National Instruments ${ }^{4}$ is used to deliver the input current, as well as, to measure the output current. The module can manage voltages between $\pm 10 \mathrm{~V}$ with a high resolution $(3.5 \mathrm{mV})$. Additionally, a custom-made electronic module that makes switching between grippers possible was built and used. All the tests and their parame-

\footnotetext{
${ }^{1}$ https://www.zeiss.com/corporate/int/home.html

2 https://www.orsayphysics.com/

3 https://sourcesup.renater.fr/www/cvlink/

4 https://www.ni.com/en-us.html
} 
ters (inputs, outputs) were performed and managed online using a single MATLAB / Simulink program and a simulation step of 0.2 seconds.

\section{Supporting Information}

Supporting Information is available from the Wiley Online Library or from the author.

\section{Acknowledgements}

We thank Blandine Guichardaz and Francois Marionnet for their technical support during this project and André Ramos for the preliminary studies.

This work has been supported by EIPHI Graduate School (ANR17-EURE-0002) and by the Bourgogne

Franche-Comté Nanofolding regional project.

\section{References}

[1] C. Hu, S. Pané, B. J. Nelson, Annual Review of Control, Robotics, and Autonomous Systems 2018, 153.

[2] M. Sitti, Nature Reviews Materials 2018, 3, 674.

[3] B. Wang, K. Kostarelos, B. J. Nelson, L. Zhang, Advanced Materials 2021, 33, 42002047.

[4] N. Liu, H. Liu, S. Zhu, H. Giessen, Nature Photonics 2009, 3, 3157.

[5] M. Hentschel, L. Wu, M. Scha“ferling, P. Bai, E. P. Li, H. Giessen, Acs Nano 2012, 6, 1110355.

[6] Y. Zhao, M. Belkin, A. Alù, Nature communications 2012, 3, 11.

[7] M. Deubel, G. Von Freymann, M. Wegener, S. Pereira, K. Busch, C. M. Soukoulis, Nature materials 2004, 3, 7444 .

[8] M. S. Rill, C. Plet, M. Thiel, I. Staude, G. Von Freymann, S. Linden, M. Wegener, Nature materials 2008, 7, 7543.

[9] T. Gissibl, S. Thiele, A. Herkommer, H. Giessen, Nature Photonics 2016, 10, 8554.

[10] G. Adam, A. Benouhiba, K. Rabenorosoa, C. Clévy, D. J. Cappelleri, Advanced Intelligent Systems 2021, 2000216.

[11] W. L. Noorduin, A. Grinthal, L. Mahadevan, J. Aizenberg, Science 2013, 340, 6134832.

[12] N. Li, P. Zhao, D. Astruc, Angewandte Chemie International Edition 2014, 53, 71756.

[13] P. X. Gao, Y. Ding, W. Mai, W. L. Hughes, C. Lao, Z. L. Wang, Science 2005, $309,57411700$.

[14] S. Xu, Z. Yan, K.-I. Jang, W. Huang, H. Fu, J. Kim, Z. Wei, M. Flavin, J. McCracken, R. Wang, et al., Science 2015, 347, 6218154.

[15] J.-H. Cho, M. D. Keung, N. Verellen, L. Lagae, V. Moshchalkov, P. Van Dorpe, D. H. Gracias, Small 2011, \%, 141943.

[16] Z. Jiang, J. He, S. A. Deshmukh, P. Kanjanaboos, G. Kamath, Y. Wang, S. K. Sankaranarayanan, J. Wang, H. M. Jaeger, X.-M. Lin, Nature materials 2015, 14, 9912.

[17] P. Zhuang, Y. Sun, L. Li, M. O. L. Chee, P. Dong, L. Pei, H. Chu, Z. Sun, J. Shen, M. Ye, et al., Advanced Materials 2020, 32, 141908072.

[18] X. Zhang, L. Medina, H. Cai, V. Aksyuk, H. D. Espinosa, D. Lopez, Advanced Materials 2020, 2005275.

[19] X. Cheng, Y. Zhang, Advanced Materials 2019, 31, 361901895. 
[20] Y. Mao, Y. Zheng, C. Li, L. Guo, Y. Pan, R. Zhu, J. Xu, W. Zhang, W. Wu, Advanced materials 2017, 29, 191606482.

[21] T. Yoshida, A. Baba, T. Asano, Japanese journal of applied physics 2005, 44, 7S 5744.

[22] W. J. Arora, H. I. Smith, G. Barbastathis, Microelectronic engineering 2007, 84, 5-8 1454.

[23] N. S. Rajput, A. Banerjee, H. Verma, Nanotechnology 2011, 22, 48485302.

[24] B. C. Park, K. Y. Jung, W. Y. Song, B.-h. O, S. J. Ahn, Advanced Materials 2006, $18,195$.

[25] C. Borschel, R. Niepelt, S. Geburt, C. Gutsche, I. Regolin, W. Prost, F.-J. Tegude, D. Stichtenoth, D. Schwen, C. Ronning, Small 2009, 5, 222576.

[26] K. Chalapat, N. Chekurov, J. Li, G. Paraoanu, Nuclear Instruments and Methods in Physics Research Section B: Beam Interactions with Materials and Atoms 2012, 272202.

[27] K. Chalapat, N. Chekurov, H. Jiang, J. Li, B. Parviz, G. Paraoanu, Advanced materials 2013, 25, 1 91.

[28] A. Cui, Z. Liu, J. Li, T. H. Shen, X. Xia, Z. Li, Z. Gong, H. Li, B. Wang, J. Li, et al., Light: Science \& Applications 2015, 4, 7 e308.

[29] Y. Mao, Y. Pan, W. Zhang, R. Zhu, J. Xu, W. Wu, Nano letters 2016, 16, 117025.

[30] X. Chen, Y. Xia, Y. Mao, Y. Huang, J. Zhu, J. Xu, R. Zhu, L. Shi, W. Wu, Micromachines 2020, 11, 195.

[31] J.-Y. Rauch, O. Lehmann, P. Rougeot, J. Abadie, J. Agnus, M. A. Suarez, Journal of Vacuum Science \& Technology A: Vacuum, Surfaces, and Films 2018, 36, 4041601.

[32] Z. Liu, H. Du, J. Li, L. Lu, Z.-Y. Li, N. X. Fang, Science advances 2018, 4, 7 eaat4436.

[33] P. Jia, K. Zuber, Q. Guo, B. C. Gibson, J. Yang, H. Ebendorff-Heidepriem, Materials Horizons 2019, 6, 51005 .

[34] A. Benouhiba, P. Rougeot, M. Ouisse, C. Clévy, N. Andreff, K. Rabenorosoa, Frontiers in Robotics and $A I$ 2019, 6122. 


\section{Table of Contents}

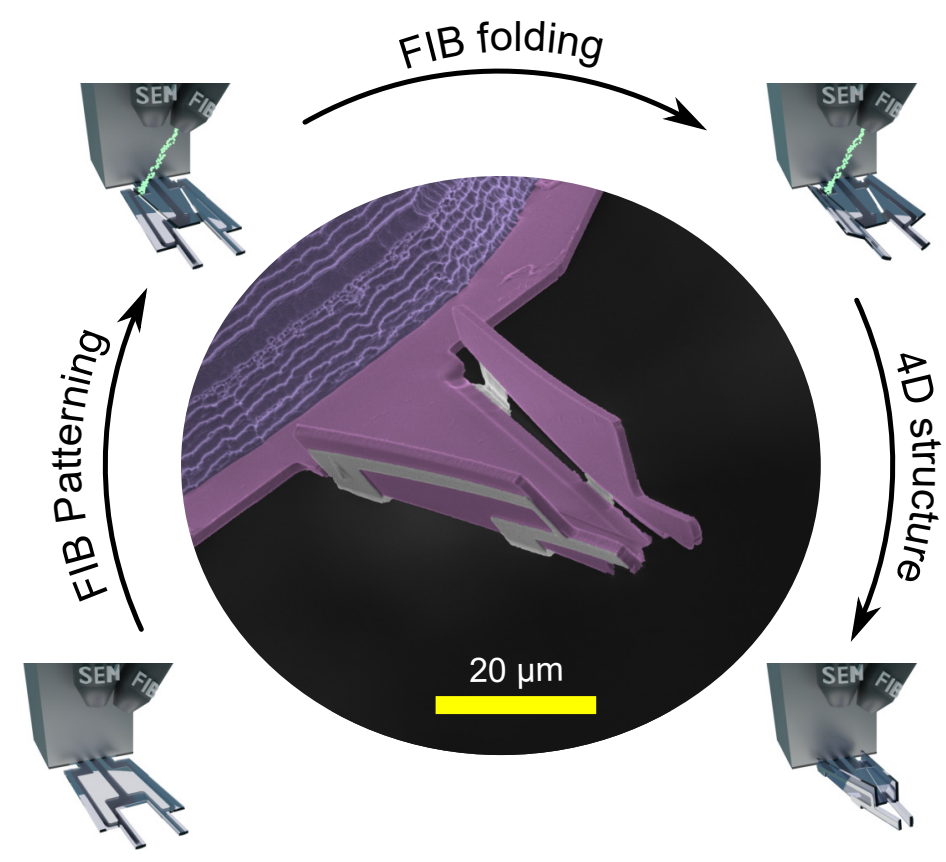

Here, a novel fabrication method for 4D nanorobotic systems via ion-induced folding is introduced. By tailoring the patterning and folding parameters, dynamic 4D microstructures with personalized properties are achieved using planar multilayer structures obtained by batch fabrication. High nanorobotic performances have been demonstrated theoretically and experimentally through a folded gripper. 